\title{
Programmable Magnetoinductive Devices
}

\author{
J. Paszkiewicz, E. Shamonina, C.J. Stevens \\ University of Oxford, Department of Engineering Science, Parks Road, Oxford OX1 3PJ, UK \\ jan.paszkiewicz@keble.ox.ac.uk
}

\begin{abstract}
A design for a programmable magnetoinductive waveguide where the impedance of each cell can be set to one of two values by an electronic signal is presented. A $1 D$ magnetoinductive waveguide using such cells is verified experimentally and used to demonstrate an electronically programmable transfer function. Extension of the concept to 2D devices and creating programmable RF systems is discussed.
\end{abstract}

\section{INTRODUCTION}

Magnetoinductive waves are slow waves propagating along arrays of resonant circuits [1]. Previous work has investigated applications of magnetoinductive devices in data [2] and power transfer [3]. By altering the impedance of each element electronically, the properties of different regions of such a metamaterial can be controlled, allowing the creation of electronically-defined RF devices such as switches, attenuators, and filters from a 1D magnetoinductive waveguide (MIW). A 2D programmable magnetoinductive surface would hypothetically allow the creation of an analogue, RF equivalent to a Field-Programmable Gate Array (FPGA), on which an entire RF system could be implemented.

\section{DESIGN OF UNIT CELLS}

As a first demonstration of a programmable MIW, it was decided to use composite unit cells with digital control of their impedance, with 'low-Z' and 'high-Z' states, referring to low and high impedance at the design frequency respectively. Each composite cell consists of a main resonator $\left(\mathrm{L}_{11}=193 \mathrm{nH}, \mathrm{C}_{1}=36 \mathrm{pF}, \mathrm{d}_{1}=20 \mathrm{~mm}\right)$ and a control resonator $\left(\mathrm{L}_{22}=38.3 \mathrm{nH}, \mathrm{C}_{2}=198 \mathrm{pF}, \mathrm{d}_{2}=12.5 \mathrm{~mm}\right)$, which are magnetically coupled $\left(\mathrm{L}_{12}=\right.$ $22.8 \mathrm{nH}$ ), as shown in Figure 1a. These are implemented as nested square printed copper coils with surfacemount components (Figure 1c). Multiple components are used in parallel to reduce the resistance of the cell.

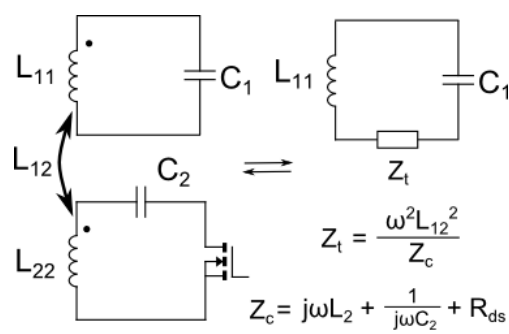

(a)



(b)

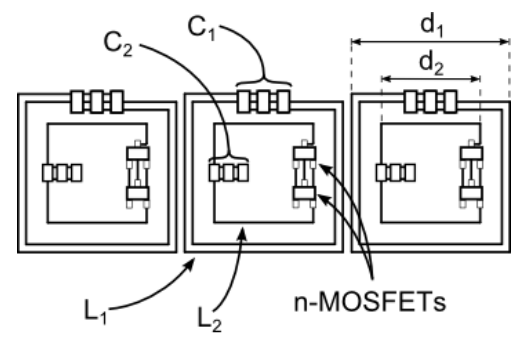

(c)

Fig. 1. Equivalent circuit of a composite digital unit cell, where $\mathrm{R}_{\mathrm{ds}}$ is the drain-source resistance of the MOSFET (a). Impedance of a single unit cell in the low- $Z$ and high- $Z$ states, referred to the main (outer) resonator, inferred from $S_{21}$ measurement using coupled non-resonant probes (b). Physical layout of unit cells on a PCB (c).

Switching the MOSFETs (type FDV303N) in the control resonator on and off corresponds to the cell becoming a system of two coupled resonators or a single resonator respectively. As the control and main resonators are nominally tuned to the same frequency, their interaction results in a high impedance at this frequency when in the high- $Z$ state, shown in Figure $1 \mathrm{~b}$. Thus, the impedance of a unit cell at its design 
frequency of $57.2 \mathrm{MHz}$ can be varied between $0.8 \Omega$ and $66 \Omega$, while maintaining a $\mathrm{Q}$ factor of 80 in the low-Z state.

\section{1D DEVICE: EXPERIMENTAL DEMONSTRATION}

To demonstrate a programmable MIW, a 1D device was created by arranging seven unit cells in a line. The state of each cell was individually controllable by applying the driving the gate-source voltage of the MOSFETs in every unit cell appropriately ( $0 \mathrm{~V}$ for low-Z, $5 \mathrm{~V}$ for high-Z). The transfer function of each cell was measured by placing an $8 \mathrm{~mm}$ diameter, non-resonant loop probe at a height of $6 \mathrm{~mm}$ below the centre of the first cell to act as the signal input. A similar probe was placed $6 \mathrm{~mm}$ above the centre line of the MIW which could be moved along the length of the MIW and acted as the output. The two probes were connected to a vector network analyser and the $S_{21}$ value was measured for different configurations. A background reading was taken without the MIW present.

The transfer function was determined by subtracting the background $S_{21}$ from the output $S_{21}$ to remove the effect of direct coupling between the two probes, then dividing the output $S_{21}$ taken at the $7^{\text {th }}$ cell by the output $\mathrm{S}_{21}$ taken at the $1^{\text {st }}$ cell, plotted in Figure 2. This effectively results in a ratio of currents between the $7^{\text {th }}$ and $1^{\text {st }}$ cells. Since the probes are sensitive to currents flowing in both the main and control resonators within a unit cell, the inferred 'current' is actually a linear combination of the two currents, dependent on the mutual inductance of each resonator with the probe.

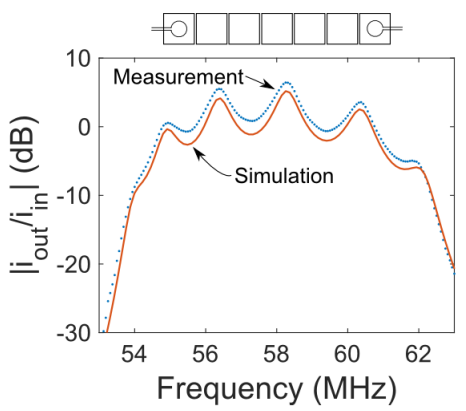

(a)

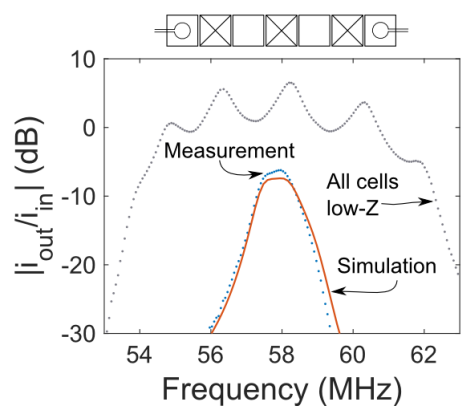

(b)

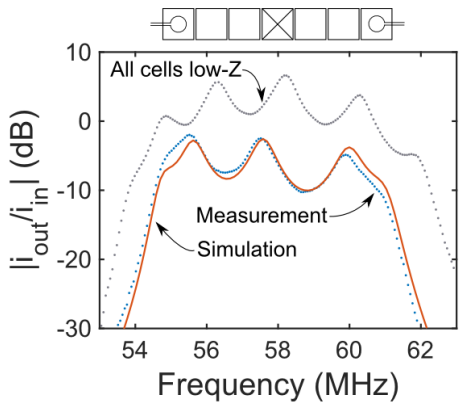

(c)

Fig. 2. Plots of the transfer function of the 7-element 1D MIW under different configurations: waveguide - all cells in low-Z state (a), Bragg reflector - cells in alternating high and low-Z states (b), attenuator - single cell in high-Z state (c). The measurement from (a) is plotted again in (b) and (c) for comparison.

The measurements show good agreement with a simulation which uses a linear circuit model for each cell and component values obtained from measurements of a single composite unit cell as well as current sheet calculations [4] of mutual inductances based on the geometry of the cells.

The transfer functions corresponding to three configurations of interest are shown in Figure 2. Figure 2a shows the case when all the cells are in the low-Z state - i.e., a normal MIW. Figure $2 b$ shows the case when every other cell is in the high-Z state, effectively doubling the cell spacing and thus reducing the coupling. This can be treated as a Bragg reflector [5] and has a narrower passband. Figure 2c shows the case when only the middle cell is in the high- $Z$ state, which introduces an impedance mismatch into the MIW, resulting in a reduction in the signal power reaching the last cell, and a change in the standing wave pattern, moving the peaks in the transfer function. These cases show the potential for programming filters and switches using 1D MIW structures.

\section{2D DEVICES}

With the concept of a 1D magnetoinductive waveguide with programmable properties having been demonstrated in the previous section, we now consider 2D systems. These allow the creation of systems with 
multiple inputs and outputs, multiple RF devices, and programmable routing between them. The simulation used to predict the behavior of the 1D MIW was extended to allow 2D arrays of arbitrary size. As an example of such behavior, a single-cell wide path of cells in the low-Z state was defined in a 10x10 array of simulated cells and a signal injected into one end of this path (Figure 3a). The simulation showed the signal following this path and reaching the other end, albeit with significant attenuation of about $20 \mathrm{~dB}$ (Figure $3 \mathrm{~b}$ ).

In order to verify this experimentally, a $4 \times 4$ array of cells has been assembled, as pictured in Figure $3 \mathrm{c}$. Measurements of this array have shown good agreement with simulations.

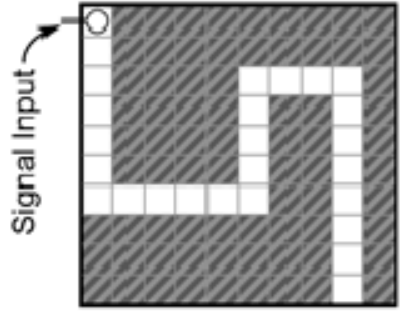

(a)

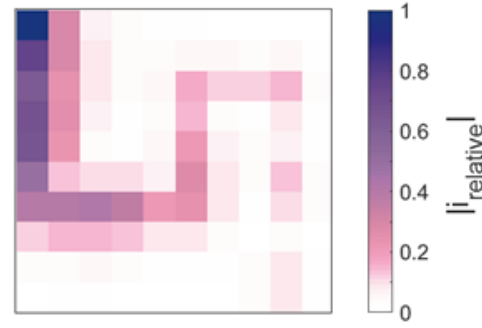

(b)

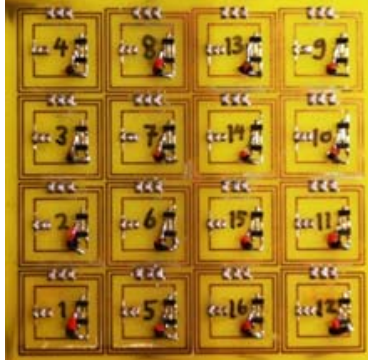

(c)

Fig. 3. 2D magnetoinductive devices: possible configuration of a $10 \times 10$ array of cells where white indicates low- $Z$ and shaded indicates high-Z (a), simulation result at $62 \mathrm{MHz}$ showing normalized current amplitude in each cell (b), photograph of a $4 \times 4$ array of cells which is being used to demonstrate a programmable $2 \mathrm{D}$ magnetoinductive device.

\section{CONCLUSIONS}

A controllable magnetoinductive element with an acceptable Q factor (80) and ratio of impedances (25 $\mathrm{dB})$ between high and low impedance states has been demonstrated using a common PCB etching process and readily available components. Using these cells, a 1D MIW with an electronically controllable transfer function can be constructed from such cells and has been verified experimentally. A 2D metasurface with an electronically programmable signal path has been shown to be feasible in simulation and has the potential to be used to create practical RF devices.

Further work includes increasing the $\mathrm{Q}$ factor of the resonators to reduce attenuation and reducing the interaction of a cell in the high- $Z$ state with the remainder of the device away from the resonant frequency. Opportunities also exist for the investigation of analogue control of the resonant frequency of each unit cell, exploration of possible devices that can be created in $1 \mathrm{D}$ and $2 \mathrm{D}$ programmable structures, as well as performance analysis and optimization of these devices.

\section{REFERENCES}

[1] E. Shamonina, V. Kalinin, K. Ringhofer and L. Solymar, "Magneto-inductive waveguide", Electron. Lett., vol. 38 , no. 8, p. 371, 2002.

[2] C. Stevens, C. Chan, K. Stamatis and D. Edwards, "Magnetic Metamaterials as 1-D Data Transfer Channels: An Application for Magneto-Inductive Waves", IEEE Transactions on Microwave Theory and Techniques, vol. 58, no. 5, pp. 1248-1256, 2010.

[3] C. Stevens, "Magnetoinductive Waves and Wireless Power Transfer", IEEE Transactions on Power Electronics, vol. 30, no. 11, pp. 6182-6190, 2015.

[4] M. Kamon, M. Ttsuk and J. White, "FASTHENRY: a multipole-accelerated 3-D inductance extraction program", IEEE Transactions on Microwave Theory and Techniques, vol. 42, no. 9, pp. 1750-1758, 1994.

[5] R. Syms, E. Shamonina and L. Solymar, "Magneto-inductive waveguide devices", IEE Proc.-Microw. Antennas Propag., vol. 153, no. 2, pp. 111-121, 2006. 